\title{
Sensitivity Analysis of Deviation Source for Fast Assembly Precision Optimization
}

\author{
Jianjun Tang, Xitian Tian, and Junhao Geng \\ Institute of CAPP \& Manufacturing Engineering Software, Northwestern Polytechnical University, Xian 710072, China \\ Correspondence should be addressed to Junhao Geng; gengjunhao@nwpu.edu.cn
}

Received 26 December 2013; Accepted 28 February 2014; Published 17 April 2014

Academic Editor: Manyu Xiao

Copyright (C) 2014 Jianjun Tang et al. This is an open access article distributed under the Creative Commons Attribution License, which permits unrestricted use, distribution, and reproduction in any medium, provided the original work is properly cited.

\begin{abstract}
Assembly precision optimization of complex product has a huge benefit in improving the quality of our products. Due to the impact of a variety of deviation source coupling phenomena, the goal of assembly precision optimization is difficult to be confirmed accurately. In order to achieve optimization of assembly precision accurately and rapidly, sensitivity analysis of deviation source is proposed. First, deviation source sensitivity is defined as the ratio of assembly dimension variation and deviation source dimension variation. Second, according to assembly constraint relations, assembly sequences and locating, deviation transmission paths are established by locating the joints between the adjacent parts, and establishing each part's datum reference frame. Third, assembly multidimensional vector loops are created using deviation transmission paths, and the corresponding scalar equations of each dimension are established. Then, assembly deviation source sensitivity is calculated by using a first-order Taylor expansion and matrix transformation method. Finally, taking assembly precision optimization of wing flap rocker as an example, the effectiveness and efficiency of the deviation source sensitivity analysis method are verified.
\end{abstract}

\section{Introduction}

In the aerospace industry, products are more and more complex, and products' precision requirements are also increasing. Precision performance of complex products is mainly guaranteed by the assembly process. The final assembly precision is affected by multiple assembly deviation sources. The degree of influence is called sensitivity. And the different sensitivities lead to the result that the difficulty of precision optimization [1] is different. Therefore, assembly precision can be optimized accurately and rapidly by analyzing each deviation source's sensitivity and reducing tolerance of the deviation source which has large sensitivity.

A typical assembly precision model requires input data including component geometry and tolerance specifications and assembly information (such as assembly sequences, locating, and clamping) to produce the desired dimensional output. Many commercial software packages exist for this purpose, such as vis VSA [2] and CE/TOL6 $\sigma$ [3], using Monte Carlo simulations. These tools are built on commonly accepted GD\&T standards [4] and adopt more recent research results, as reported in [5-7]. The basic assumption is that the product is comprised of rigid bodies.

Tolerance design in computer aided process planning needs to obtain an appropriate set of manufacturing tolerances for the various manufacturing operations involved, considering process capability of the machines and manufacturing allowance for each operation in succession. Aiming at drawbacks of tolerance design [8-11], a few authors proposed a series of optimization methods [12-15]. Chen and Chung [16] introduced a model to determine the inspection precision and the optimal number of repeated measurements in order to maximize the net expected profit per item. Kannan and Jayabalan [17] used a Genetic Algorithm to solve the problem by generating six partitions for each component (subassembly). One kind of method used small displacements torsor (SDT) to model the process planning [18].

Assembly precision optimization [19-22] is an important means to ensure product quality. Assembly deviation source sensitivity analysis [23-26] provides critical information for assembly precision optimization. ANSELMETTI [27] 
proposed the concept of deviation sources. Deviation transmission mechanism was analyzed, but the effect of deviation source sensitivity to cumulative deviation was not considered in the multidimensional environment. Mansuy et al. [28] introduced segmented geometric elements along the three-dimensional vector direction and then calculated the sensitivity and predicted the precision. Laperrière and ElMaraghy [29] proposed a method of tolerance analysis by describing the tolerance vector with six scalar equations in three-dimensional space. But it did not consider the role of assembly datum in the process of deviation transmission.

Because the physical world is predominantly nonlinear, Monte Carlo simulation is naturally the most accurate and sometimes the only method for tolerance analysis for a generic assembly product. However, computational inefficiency from Monte Carlo simulations hinders many advanced features such as assembly precision and sequence optimization, which all require a significant number of analysis iterations.

For most engineering applications, including the wing assembly, the nonlinear kinematic relations can be approximated by linear models through a first-order Taylor series expansion. Therefore, developing an efficient linear deviation source sensitivity analysis method is both imperative and feasible. In multidimensional space, for a comprehensive sensitivity analysis of mutual coupled deviation source, an assembly deviation source sensitivity calculation method is proposed based on multidimensional vector loops. Combining the characteristic of complex product assembly, multidimensional vector loops are built. Based on the loops, the sensitivity of each deviation source is calculated by reducedorder operation.

This paper is organized as follows. Section 2 gives the principle of assembly precision optimization. Section 3 proposes a method of deviation source sensitivity analysis. Section 4 presents a computational example to demonstrate the method. Section 5 comes up with a summary for this paper.

\section{Principle of Assembly Precision Optimization}

Tolerance analysis methods have the worst case and RSS method, shown as follows [21]:

$$
\begin{gathered}
t_{\mathrm{wc}}=\sum_{i=1}^{n}\left|a_{i} t_{i}\right|, \\
t_{\mathrm{rss}}=\sqrt{\sum_{i=1}^{n}\left(a_{i} t_{i}\right)^{2}} .
\end{gathered}
$$

Assembly precision optimization is a process of solving the minimum value of assembly deviation value. Another way to determine value of sensitivity, $a_{i}$, is developed for fast assembly precision optimization:

$$
\begin{gathered}
\min t_{\mathrm{wc}}=\min \sum_{i=1}^{n}\left|a_{i} t_{i}\right|, \\
\min t_{\mathrm{rss}}=\sqrt{\min \sum_{i=1}^{n}\left(a_{i} t_{i}\right)^{2} .}
\end{gathered}
$$

According to (3), $a_{i}$ determines the influence of $t_{i}$ to $t_{\mathrm{wc}}$ and $t_{\mathrm{rss}}$. In process of assembly precision optimization, by determining the $a_{i}$, the deviation source, which needs to be optimized, can be quickly determined; then $\min t_{\mathrm{wc}}$ and min $t_{\text {rss }}$ can be obtained. So the most important thing of assembly precision optimization is calculating each of the deviation source sensitivities.

In one-dimensional space, $a_{i}$ is defined as \pm 1 (increases ring $a_{i}=+1$, decreases ring $a_{i}=-1$ ). In multidimensional space, because of the uncertainty of deviation vector direction, $a_{i}$ can be dramatically magnified. So every deviation source sensitivity $a_{i}$ should be calculated. Determining which $a_{i}$ is bigger, the corresponding deviation source is the one which needs to be reduced. By decreasing value of deviation source, assembly precision optimization is realized effectively.

The result of assembly deviation accumulation expresses the variable between actual assembly dimension and design dimension. The sensitivities are solved by taking partial derivatives with respect to each variable.

Assume that $\left\{y_{1}, y_{2}, \ldots, y_{n}\right\}$ are the corresponding part's dimensions of deviation sources, and a function $\Theta\left(y_{1}, y_{2}, \ldots, y_{n}\right)$ expresses value of assembly dimension. The sensitivity of $\Theta$ with respect to $y_{i}$ is

$$
a_{i}=\left.\frac{\partial \Theta}{\partial y_{i}}\right|_{\text {NominalValues }} .
$$

The partial derivative at the nominal values of each variable is evaluated. And the nominal value for each variable is the center of a tolerance range, or the value of the dimension when the tolerances are equal bilaterally.

\section{Deviation Source Sensitivity Analysis Method}

In the process of complicated product assembly, a variety of joint types are required to describe the mating parts' contact points. Different joint types form different kinds of deviation source couplings. And different couplings lead to different results of deviation transmission. Now deviation transmission path, vector loop equations, and sensitivity calculation method are discussed, respectively.

\subsection{Deviation Transmission Path Based on Datum Reference} Frames. Assembly parts are located through the locating datum in the process of product assembly. In the process of forming deviation transmission path, part's locating datum is called datum reference frame (DRF). DRF is mainly used for locating every needed feature of a part. Datum path (DP) is a path from the joint to the DRF which is connected with nominal dimension vectors. A deviation transmission path of two constrained parts is along a DP and through a joint. 
It must obey certain modeling rules as it passes through a part. It must

(1) enter a part through a joint;

(2) follow the DP to the DRF in the part;

(3) follow a second DP leading to another joint;

(4) exit to the next adjacent part from the joint in the assembly.

The deviation transmission path is illustrated in Figure 1. $J$ (Part1, Part2) is the joint of Part1 and Part2. There are four DPs in Partl and Part2 $\left(\mathrm{DP}_{11}, \mathrm{DP}_{12}, \mathrm{DP}_{21}\right.$, and $\left.\mathrm{DP}_{22}\right)$. They are created by dimension vectors $(a, b, c, d, e, f, g$, and $h)$. All the dimension vectors are important for sensitivity analysis.

3.2. Dimension Vector Loops and Vector Equations. According to product's assembly sequence and locating, a dimension vector loop is formed by connecting all the deviation transmission paths. Dimension vector loop is divided into closed loop and opened loop. Closed loop describes relations between nominal dimensions of parts and assembly dimensions. Opened loop describes the influence of parts' nominal dimensions to the key characteristics in the assembly. Modeling rules for dimension vector loops include the following.

(1) Loops must pass through every part and every joint in the assembly.

(2) A single vector loop passes through the same joint no more than once, but it may start and end in the same part.

(3) If a vector loop includes the exact same dimension twice, in opposite directions, the dimension must be omitted.

(4) For an assembly, the number of closed loop $L$ can be expressed as

$$
L=J-P+1,
$$

where $J$ is the number of joints and $P$ is the number of parts.

Assuming that an assembly contains part dimensions $\left\{x_{1}, x_{2}, \ldots, x_{n}\right\}$ and assembly dimensions $\left\{U_{1}, U_{2}, \ldots, U_{m}\right\}$, multidimensional vector closed loops can be expressed as

$$
h_{D}=D\left(x_{1}, x_{2}, \ldots, x_{n}, U_{1}, U_{2}, \ldots, U_{m}\right)=0,
$$

where $D$ is the vector direction of dimensions; for example, in three-dimensional space, $D=(u, v, w, \alpha, \beta, \gamma)$, and, in twodimensional space, $D=(x, y, \theta)$. The parameters $(u, v, w, x$, and $y)$ are location parameters of a vector. The parameters $(\alpha, \beta, \gamma$, and $\theta)$ are direction parameters of a vector.

Multidimensional vector opened loops can be expressed as

$$
\text { Gap }=\Gamma\left(x_{1}, x_{2}, \ldots, x_{n}, U_{1}, U_{2}, \ldots, U_{m}\right),
$$

where $\Gamma$ is the vector direction along the opened loop distance.

By (4), the explicit expression of $x$ and $U$ should be calculated. But (6) and (7) are nonlinear and implicit. They contain products and trigonometric functions of the variables. So certain mathematical methods are needed for sensitivity analysis.
3.3. Order Reduction of Vector Loop Equations. The assembly deviation describes a small variable of dimension. For explicit expression of $x$ and $U$, first-order Taylor's series expansion [30] of (6) and (7) is used. The following equations show the explicit expression for closed loop and opened loop:

$$
\begin{aligned}
\delta h_{D}= & \frac{\partial h_{D}}{\partial x_{1}} \delta x_{1}+\cdots+\frac{\partial h_{D}}{\partial x_{n}} \delta x_{n} \\
& +\frac{\partial h_{D}}{\partial U_{1}} \delta U_{1}+\cdots+\frac{\partial h_{D}}{\partial U_{n}} \delta U_{n}=0, \\
\delta \mathrm{Gap}= & \frac{\partial \mathrm{Gap}}{\partial x_{1}} \delta x_{1}+\cdots+\frac{\partial \mathrm{Gap}}{\partial x_{n}} \delta x_{n} \\
& +\frac{\partial \mathrm{Gap}}{\partial U_{1}} \delta U_{1}+\cdots+\frac{\partial \mathrm{Gap}}{\partial U_{n}} \delta U_{n} .
\end{aligned}
$$

Equations (8) and (9) may be written in matrix form and solved for the deviation source sensitivities by matrix algebra. Equation (8) can be expressed in matrix form as follows:

$$
[A][\delta x]+[B][\delta U]=0 .
$$

The multidimensional vector opened loop scalar equation (9) can be expressed in matrix form as follows:

$$
[\delta \mathrm{Gap}]=[C][\delta x]+[E][\delta U],
$$

where $[A]$ is the partial derivative matrix of a closed loop scalar equation to the part dimension variables; $[B]$ is the partial derivative matrix of a closed loop scalar equation to the assembly dimension variables; $[\delta x]$ is the vector of small variations in the part dimensions; $[\delta U]$ is the vector of small variations in the assembly dimensions; $[C]$ is the partial derivative matrix of an opened loop scalar equation to the part dimension variables; $[E]$ is the partial derivative matrix of an opened loop scalar equation to the assembly dimension variables; and $[\delta \mathrm{Gap}]$ is the vector of variations in the assembly key characteristics.

By matrix transformation, (10) and (11) are expressed in the following form:

$$
\begin{gathered}
{[\delta U]=-\left[B^{-1} A\right][\delta x],} \\
{[\delta \text { Gap }]=\left[C-E B^{-1} A\right][\delta x] .}
\end{gathered}
$$

The matrix $-\left[B^{-1} A\right]$ is the sensitivity matrix of assembly dimension variables with respect to deviation source dimension variables. The matrix $\left[C-E B^{-1} A\right]$ is the sensitivity matrix of assembly key characteristics dimension variables with respect to deviation source dimension variables.

\section{Computational Experiment}

Taking assembly deviation source sensitivity analysis of wing flap rocker as a research example, the deviation transmission paths and dimension vector loops are established. The main influence factors of assembly precision optimization are analyzed based on calculating deviation source sensitivity. 


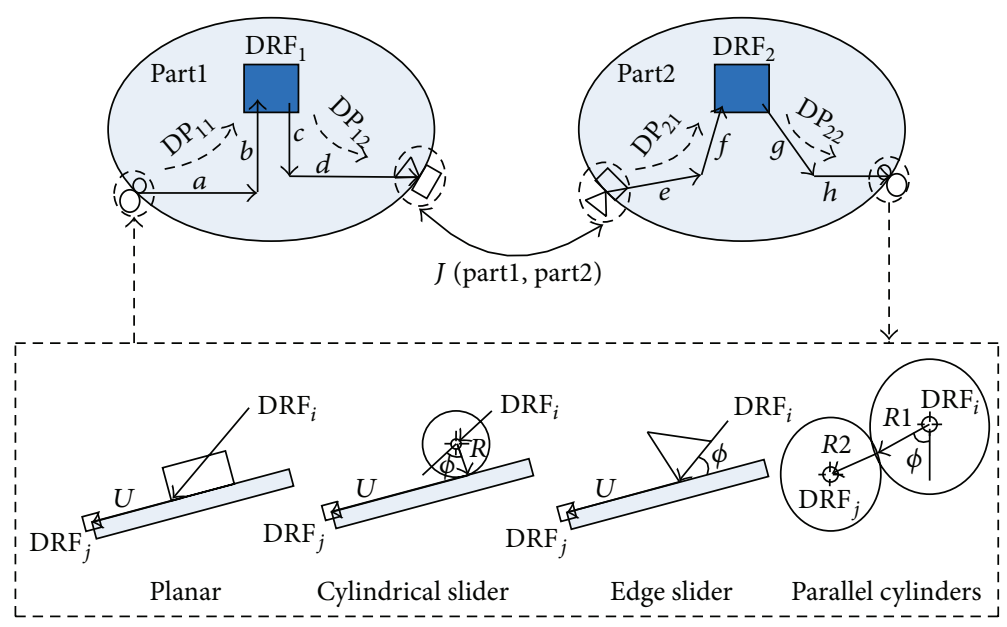

FIGURE 1: Deviation transmission path formed by four kinds of joint types.

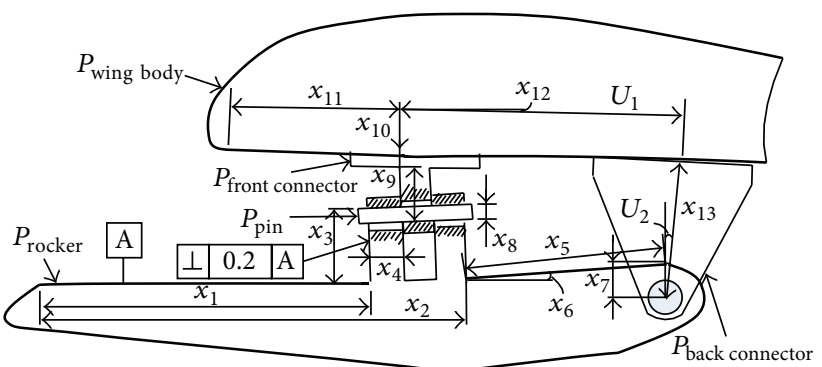

FIGURE 2: A profile of wing flap rocker assembly.

Wing flap rocker mainly contains five components: $P_{\text {wing body }}, P_{\text {front connector }}, P_{\text {back connector }}, P_{\text {rocker }}$, and $P_{\text {pin }}$, shown in Figure 2. The deviation sources are $x_{1}, x_{2}, \ldots, x_{13}$ and $\perp|0.2| \mathrm{A}$. All the deviation sources dimensions' nominal values and tolerances are shown in Table 1.

The process of wing flap rocker assembly deviation source sensitivity analysis based on dimension vector loops is illustrated in Figure 3.

First of all, DPs are established. According to assembly constraints, the connection types and joints $\left(J\left(P_{\text {rocker }}\right.\right.$, $\left.P_{\text {pin }}\right), \quad J\left(P_{\text {pin }}, \quad P_{\text {front connector }}\right), \quad J\left(P_{\text {front connector }}, \quad P_{\text {wing body }}\right)$, $J\left(P_{\text {wing body }}, \quad P_{\text {back connector }}\right)$, and $\left.J\left(P_{\text {back connector }}, P_{\text {rocker }}\right)\right)$ in the assembly are located; DRFs $\left(\mathrm{DRF}_{\text {rocker }}, \mathrm{DRF}_{\text {pin }}\right.$, $\mathrm{DRF}_{\text {front connector }}, \mathrm{DRF}_{\text {wing body, }}$, and $\left.\mathrm{DRF}_{\text {back connector }}\right)$ are defined based on factors such as component design references and assembly locating datum; DPs from joints to DRFs are created along the nominal dimension vector directions, as shown in Figure 4.

Then, the deviation transmission paths and multidimensional vector loops are created based on the DPs, as shown in Figure 5. Figure 5(a) shows assembly deviation transmission vector closed loop. Because of the $\perp|0.2| \mathrm{A}, a_{14}$ becomes a deviation source. Figure 5(b) shows assembly deviation transmission vector opened loop. Gap means the distance from $\mathrm{DRF}_{\text {wing body }}$ to $x_{1}$.

Finally, (13) is generated according to the dimensionality $D=\{x, y, \theta\}$. The parameters $\delta x, \delta U, A, B, C$, and $E$ are solved by first-order Taylor's series expansion. The parameters are shown in (14). Sensitivities are solved by matrix operation, as shown in Table 2:

$$
\begin{aligned}
h_{x}= & x_{1} \cos (0)+x_{3} \cos \left(x_{14}\right)+x_{4} \cos (0)+x_{8} \cos \left(-U_{3}\right) \\
& +x_{9} \cos (90)+x_{10} \cos (90)+U_{1} \cos \left(-x_{12}\right) \\
& +x_{13} \cos \left(-U_{2}-90\right)+x_{7} \cos (90) \\
& +x_{5} \cos \left(-180+x_{6}\right)+x_{2} \cos (-180)=0, \\
h_{y}= & x_{1} \sin (0)+x_{3} \sin \left(a_{14}\right)+x_{4} \sin (0)+x_{8} \sin \left(-U_{3}\right) \\
& +x_{9} \sin (90)+x_{10} \sin (90)+U_{1} \sin \left(-x_{12}\right) \\
& +x_{13} \sin \left(-U_{2}-90\right)+x_{7} \sin (90) \\
& +x_{5} \sin \left(-180+a_{6}\right)+x_{2} \sin (-180)=0, \\
h_{\theta}= & 0+x_{14}-90-U_{3}+180-\left(90+x_{12}\right) \\
& -90-\left(180-U_{2}\right)+90-x_{6}+180=0,
\end{aligned}
$$


TABLE 1: The deviation sources dimensions' nominal values and tolerances.

\begin{tabular}{lccccccccc}
\hline Dimension parameter & $x_{1} / \mathrm{mm}$ & $x_{2} / \mathrm{mm}$ & $x_{3} / \mathrm{mm}$ & $x_{4} / \mathrm{mm}$ & $x_{5} / \mathrm{mm}$ & $x_{6} / \operatorname{deg}$ & $x_{7} / \mathrm{mm}$ & $x_{8} / \mathrm{mm}$ & $x_{9} / \mathrm{mm}$ \\
\hline Nominal value & 260 & 320 & 42 & 20 & 221 & 9 & 28 & 10 & 30 \\
Tolerance & \pm 0.3 & \pm 0.3 & \pm 0.3 & \pm 0.3 & \pm 0.3 & \pm 0.5 & \pm 0.3 & \pm 0.3 & \pm 0.3 \\
\hline Dimension parameter & $x_{10} / \mathrm{mm}$ & $x_{11} / \mathrm{mm}$ & $x_{12} / \mathrm{deg}$ & $x_{13} / \mathrm{mm}$ & $x_{14} / \mathrm{deg}$ & $U_{1} / \mathrm{mm}$ & $U_{2} / \mathrm{deg}$ & $U_{3} / \mathrm{deg}$ & \\
\hline Nominal value & 10 & 150 & 6 & 80 & 90 & 251 & 15 & 90 & \pm 1 \\
Tolerance & \pm 0.3 & \pm 0.3 & \pm 0.5 & \pm 0.3 & \pm 0.2 & \pm 0.5 & \pm 1 & \pm 1 \\
\hline
\end{tabular}

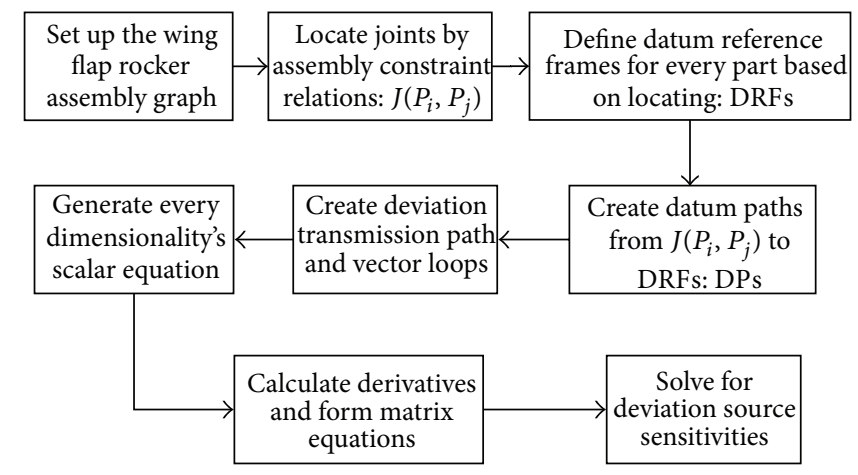

FIGURE 3: The process of assembly deviation source sensitivity analysis.

$$
\begin{aligned}
& \text { Gap }=\left(x_{11}+U_{1}\right) \sin \left(-x_{12}\right)+x_{13} \sin \left(-U_{2}-90\right) \\
& +x_{7} \sin (90)+x_{5} \sin \left(-180+x_{6}\right) \text {. } \\
& {[A]=\left[\begin{array}{llll}
\frac{\partial h_{x}}{\partial x_{1}} & \frac{\partial h_{x}}{\partial x_{2}} & \cdots & \frac{\partial h_{x}}{\partial x_{14}} \\
\frac{\partial h_{y}}{\partial x_{1}} & \frac{\partial h_{y}}{\partial x_{2}} & \cdots & \frac{\partial h_{y}}{\partial x_{14}} \\
\frac{\partial h_{\theta}}{\partial x_{1}} & \frac{\partial h_{\theta}}{\partial x_{2}} & \cdots & \frac{\partial h_{\theta}}{\partial x_{14}}
\end{array}\right]} \\
& {[B]=\left[\begin{array}{lll}
\frac{\partial h_{x}}{\partial U_{1}} & \frac{\partial h_{x}}{\partial U_{2}} & \frac{\partial h_{x}}{\partial U_{3}} \\
\frac{\partial h_{y}}{\partial U_{1}} & \frac{\partial h_{y}}{\partial U_{2}} & \frac{\partial h_{y}}{\partial U_{3}} \\
\frac{\partial h_{\theta}}{\partial U_{1}} & \frac{\partial h_{\theta}}{\partial U_{2}} & \frac{\partial h_{\theta}}{\partial U_{3}}
\end{array}\right]} \\
& {[C]=\left[\begin{array}{llll}
\frac{\partial \mathrm{Gap}}{\partial x_{1}} & \frac{\partial \mathrm{Gap}}{\partial x_{2}} & \cdots & \frac{\partial \mathrm{Gap}}{\partial x_{14}}
\end{array}\right] \text {, }} \\
& {[E]=\left[\begin{array}{lll}
\frac{\partial \mathrm{Gap}}{\partial U_{1}} & \frac{\partial \mathrm{Gap}}{\partial U_{2}} & \frac{\partial \mathrm{Gap}}{\partial U_{3}}
\end{array}\right]} \\
& {[\delta x]=\left[\begin{array}{llll}
\delta x_{1} & \delta x_{2} & \cdots & \delta x_{14}
\end{array}\right]^{\mathrm{T}} \text {, }} \\
& {[\delta U]=\left[\begin{array}{lll}
\delta U_{1} & \delta U_{2} & \delta U_{3}
\end{array}\right]^{\mathrm{T}} \text {. }}
\end{aligned}
$$

As shown in Table 2, the greatest impact on the assembly precision is the deviation sources $x_{6}, x_{12}$, and $x_{14}$. The angle dimensions $x_{6}, x_{12}$, and $x_{14}$ correspond to length dimensions $x_{5}, U_{1}$, and $x_{3}$. It can be seen that the larger the length dimension, the greater the sensitivity of the corresponding angle.

According to (1), assembly precision $\delta U_{2}=0.5394$.

The first assembly precision optimization method is that the tolerances of deviation sources $x_{1}, x_{2}, x_{3}, x_{4}, x_{5}, x_{7}, x_{8}$, $x_{9}, x_{10}, x_{11}, x_{13}$, and $x_{14}$ are reduced by $50 \%$. An optimized assembly precision is obtained; $\delta U_{2}=0.3940$.

The second assembly precision optimization method is that the tolerances of deviation sources $x_{6}$ and $x_{12}$ are reduced by $50 \%$. An optimized assembly precision is obtained; $\delta U_{2}=$ 0.2533 . So the goal of assembly precision optimization is the tolerances of deviation sources $x_{6}$ and $x_{12}$.

The results indicate that it would be more conducive to optimize the assembly precision by reducing the deviations which have large sensitivity.

\section{Conclusions}

This paper presents an approach for fast assembly precision optimization of complex products based on deviation sources sensitivities analysis. The joints between the adjacent parts and each part's datum reference frame are defined for creating deviation transmission paths and multidimensional dimension vector loops. Sensitivity calculations of assembly deviation source are established by linearizing all the multidimensional vector loop scalar equations which can be gotten using first-order Taylor's series expansion and matrix algebra.

In practice, we find that the sensitivity of deviation source is not always +1 and -1 . In the multidimensional space, sensitivity of deviation source is enlarged dramatically under certain conditions. If a list of deviation sources has the same vector directions, the sensitivities of an assembly dimension to the deviation sources are the same. If the vector direction 


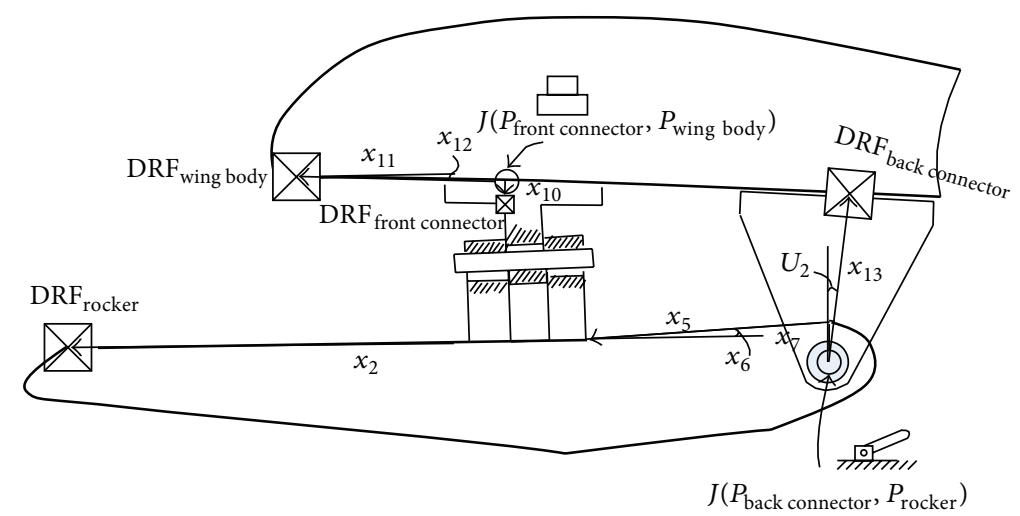

Figure 4: DRFs and DPs.

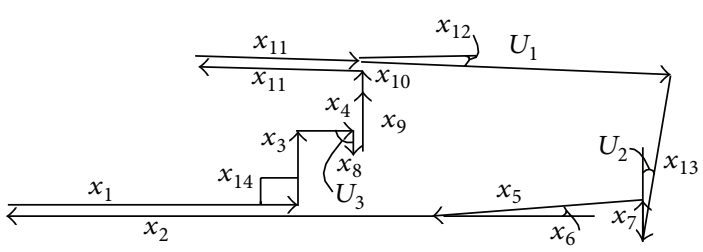

(a) The closed loop

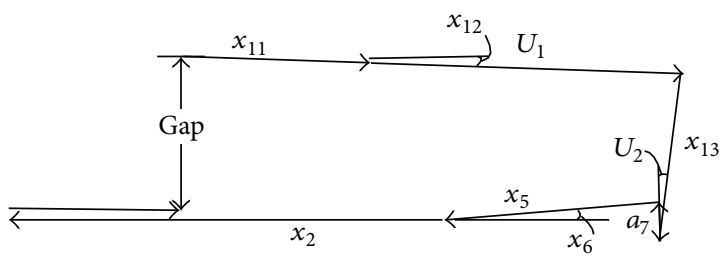

(b) The opened loop

FIgURE 5: Deviation transmission vector loops.

TABLE 2: The value of deviation source sensitivities.

\begin{tabular}{|c|c|c|c|c|c|c|c|c|c|c|c|c|c|c|}
\hline & $x_{1}$ & $x_{2}$ & $x_{3}$ & $x_{4}$ & $x_{5}$ & $x_{6}$ & $x_{7}$ & $x_{8}$ & $x_{9}$ & $x_{10}$ & $x_{11}$ & $x_{12}$ & $x_{13}$ & $x_{14}$ \\
\hline Sensitivity of $U_{1}$ & -1.8 & 1.8 & -7.9 & -1.8 & 3.1 & 1644 & -7.9 & 7.9 & -7.9 & -7.9 & 0 & 2005 & 8.1 & 99.3 \\
\hline Sensitivity of $U_{2}$ & 0 & 0 & -0.1 & 0 & 0 & 18.8 & -0.1 & 0.1 & -0.1 & -0.1 & 0 & 22.2 & 0.1 & 0.5 \\
\hline Sensitivity of $U_{3}$ & 0 & 0 & -0.1 & 0 & 0 & 17.8 & -0.1 & 0.1 & -0.1 & -0.1 & 0 & 21.2 & 0.1 & 1.5 \\
\hline Sensitivity of Gap & 0 & 0 & -1 & 0 & 0 & 0 & 0 & 1 & -1 & -1 & -0.1045 & -149 & 0 & 0 \\
\hline
\end{tabular}

of an assembly dimension and a deviation source is the same, the sensitivity of the assembly dimension to the deviation source is +1 . If the vector direction of an assembly dimension and a deviation source is opposite, the sensitivity of the assembly dimension to the deviation source is -1 . If the vector direction of an assembly dimension and a deviation source is perpendicular, the sensitivity of the assembly dimension to the deviation source is 0 .

Deviation source sensitivity is an important indicator of assembly precision optimization in the aerospace industry. To further improve the flexibility of our approach, four kinds of joint types and multidimensional vector loops are used. Considering the deviation source sensitivity analysis of complex product assembly, our future work also includes improving the algorithm by providing a more traceable and robust method.

\section{Conflict of Interests}

The authors declare that there is no conflict of interests regarding the publication of this paper.

\section{Acknowledgments}

This work was supported by the National Natural Science Foundation of China under Grant no. 51105313 and the Doctorate Foundation of Northwestern Polytechnical University under Grant no. CX201313.

\section{References}

[1] S. Shin, P. Kongsuwon, and B. R. Cho, "Development of the parametric tolerance modeling and optimization schemes and cost-effective solutions," European Journal of Operational Research, vol. 207, no. 3, pp. 1728-1741, 2010.

[2] Z. Shen, "Tolerance analysis with EDS/VisVSA," Journal of Computing and Information Science in Engineering, vol. 3, no. 1, pp. 95-99, 2003.

[3] CE/TOL6 $\sigma$, Sigmetrix LLC, http://www.sigmetrix.com/.

[4] American Society of Mechanical Engineers, ANSI/ASME Y14.5M-1994, Dimensioning and tolerancing, 1994.

[5] Y. Wu, J. J. Shah, and J. K. Davidson, "Computer modeling of geometric variations in mechanical parts and assemblies," Journal of Computing and Information Science in Engineering, vol. 3, no. 1, pp. 54-63, 2003. 
[6] P. K. Singh, S. C. Jain, and P. K. Jain, "Advanced optimal tolerance design of mechanical assemblies with interrelated dimension chains and process precision limits," Computers in Industry, vol. 56, no. 2, pp. 179-194, 2005.

[7] A. J. Qureshi, J.-Y. Dantan, V. Sabri, P. Beaucaire, and N. Gayton, "A statistical tolerance analysis approach for overconstrained mechanism based on optimization and Monte Carlo simulation," CAD Computer Aided Design, vol. 44, no. 2, pp. 132-142, 2012.

[8] Y. Zhang, Z. Li, J. Gao, J. Hong, F. Villecco, and Y. Li, "A method for designing assembly tolerance networks of mechanical assemblies," Mathematical Problems in Engineering, vol. 2012, Article ID 513958, 26 pages, 2012.

[9] G. Zhang, "Simultaneous tolerancing for design and manufacturing," International Journal of Production Research, vol. 34, no. 12, pp. 3361-3382, 1996.

[10] E. A. Lehtihet, S. Ranade, and P. Dewan, "Comparative evaluation of tolerance control chart model," International Journal of Production Research, vol. 38, no. 7, pp. 1539-1556, 2000.

[11] Y. S. Hong and T.-C. Chang, "A comprehensive review of tolerancing research," International Journal of Production Research, vol. 40, no. 11, pp. 2425-2459, 2002.

[12] H. P. Peng, X. Q. Jiang, and X. J. Liu, "Concurrent optimal allocation of design and process tolerances for mechanical assemblies with interrelated dimension chains," International Journal of Production Research, vol. 46, no. 24, pp. 6963-6979, 2008.

[13] F. W. Ciarallo and C. C. Yang, "Optimization of propagation in interval constraint networks for tolerance design," in Proceedings of the IEEE International Conference on Systems, Man, and Cybernetics, pp. 1924-1929, October 1997.

[14] B.-W. Cheng and S. Maghsoodloo, "Optimization of mechanical assembly tolerances by incorporating Taguchi's quality loss function," Journal of Manufacturing Systems, vol. 14, no. 4, pp. 264-276, 1995.

[15] S. Jung, D.-H. Choi, B.-L. Choi, and J. H. Kim, "Tolerance optimization of a mobile phone camera lens system," Applied Optics, vol. 50, no. 23, pp. 4688-4700, 2011.

[16] S.-L. Chen and K.-J. Chung, "Selection of the optimal precision level and target value for a production process: the lowerspecification-limit case," IIE Transactions, vol. 28, no. 12, pp. 979-985, 1996.

[17] S. M. Kannan and V. Jayabalan, "A new grouping method for minimizing the surplus parts in selective assembly," Quality Engineering, vol. 14, no. 1, pp. 65-75, 2001.

[18] F. Villeneuve, O. Legoff, and Y. Landon, "Tolerancing for manufacturing: a three-dimensional model," International Journal of Production Research, vol. 39, no. 8, pp. 1625-1648, 2001.

[19] S. Xu and J. Keyser, "Geometric computation and optimization on tolerance dimensioning," Computer-Aided Design, vol. 46, pp. 129-137, 2014.

[20] R. Musa, J.-P. Arnaout, and F. Frank Chen, "Optimizationsimulation-optimization based approach for proactive variation reduction in assembly," Robotics and Computer-Integrated Manufacturing, vol. 28, no. 5, pp. 613-620, 2012.

[21] S. H. Huang, Q. Liu, and R. Musa, "Tolerance-based process plan evaluation using Monte Carlo simulation," International Journal of Production Research, vol. 42, no. 23, pp. 4871-4891, 2004.

[22] M. V. Raj, S. S. Sankar, and S. G. Ponnambalam, "Optimization of assembly tolerance variation and manufacturing system efficiency by using genetic algorithm in batch selective assembly,"
International Journal of Advanced Manufacturing Technology, vol. 55, no. 9-12, pp. 1193-1208, 2011.

[23] C. C. Yang and V. N. A. Naikan, "Optimum tolerance design for complex assemblies using hierarchical interval constraint networks," Computers and Industrial Engineering, vol. 45, no. 3, pp. 511-543, 2003.

[24] P. K. Singh, S. C. Jain, and P. K. Jain, "Concurrent optimal adjustment of nominal dimensions and selection of tolerances considering alternative machines," CAD Computer Aided Design, vol. 38, no. 10, pp. 1074-1087, 2006.

[25] W. Cai, "A new tolerance modeling and analysis methodology through a two-step linearization with applications in automotive body assembly," Journal of Manufacturing Systems, vol. 27, no. 1, pp. 26-35, 2008.

[26] C. C. Yang and V. N. Achutha Naikan, "Optimum design of component tolerances of assemblies using constraint networks," International Journal of Production Economics, vol. 84, no. 2, pp. 149-163, 2003.

[27] B. Anselmetti, "Generation of functional tolerancing based on positioning features," CAD Computer Aided Design, vol. 38, no. 8, pp. 902-919, 2006.

[28] M. Mansuy, M. Giordano, and P. Hernandez, "A new calculation method for the worst case tolerance analysis and synthesis in stack-type assemblies," CAD Computer Aided Design, vol. 43, no. 9, pp. 1118-1125, 2011.

[29] L. Laperrière and H. ElMaraghy, "Tolerance analysis and synthesis using jacobian-transforms," CIRP-Annals, vol. 49, no. 1, pp. 359-362, 2000.

[30] X. Huang and Y. Zhang, "Probabilistic approach to system reliability of mechanism with correlated failure models," Mathematical Problems in Engineering, vol. 2012, Article ID 465853, 11 pages, 2012. 


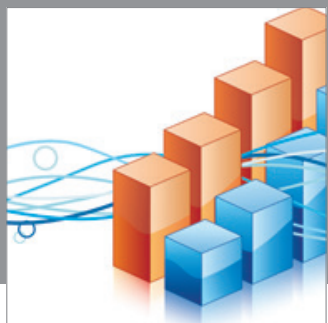

Advances in

Operations Research

mansans

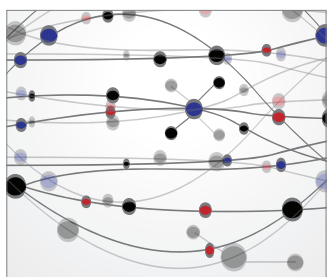

The Scientific World Journal
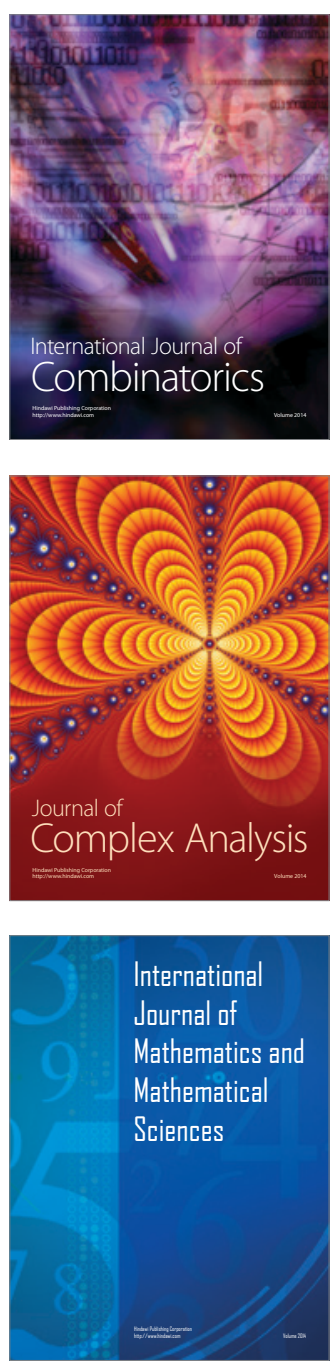
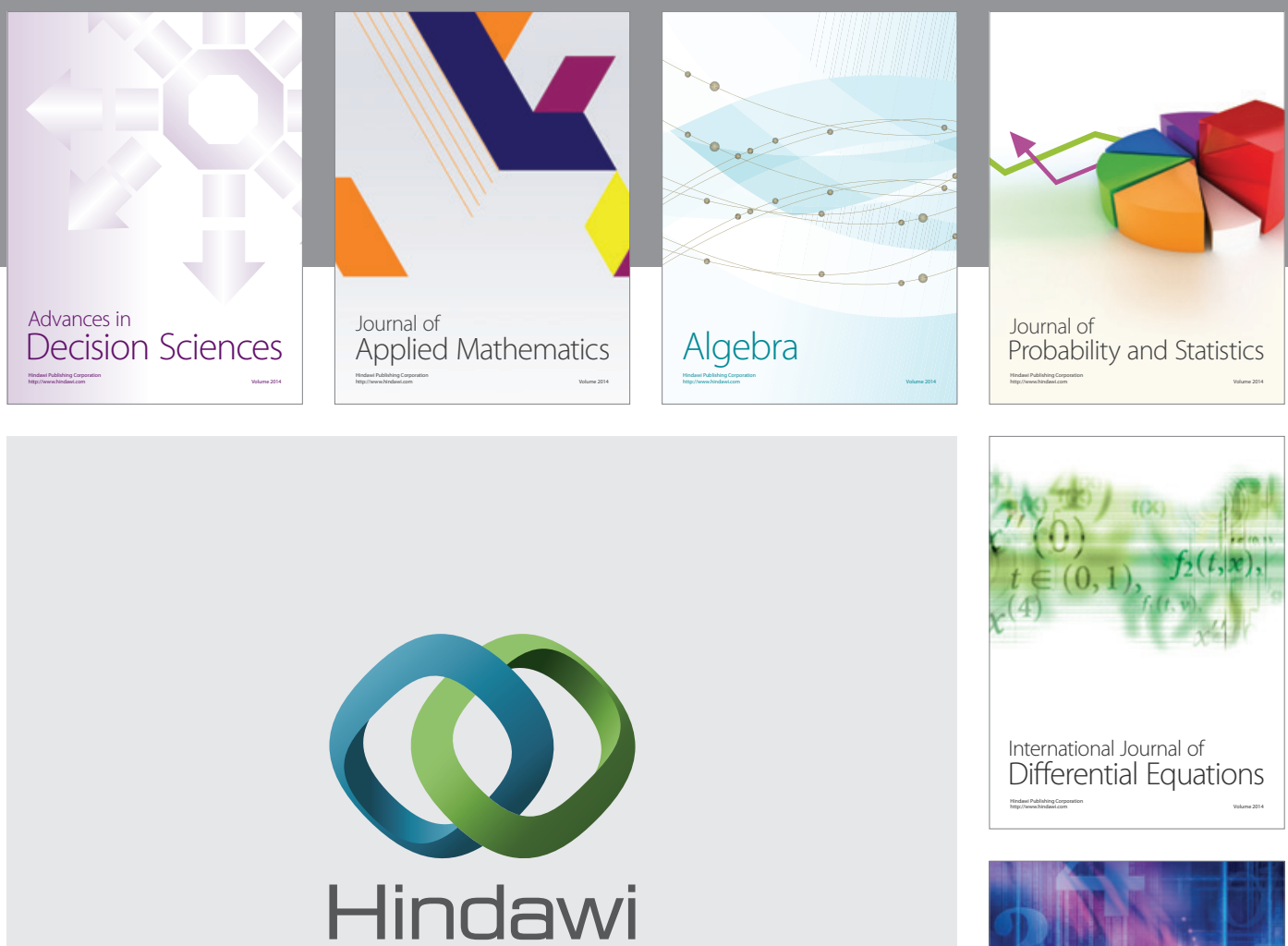

Submit your manuscripts at http://www.hindawi.com
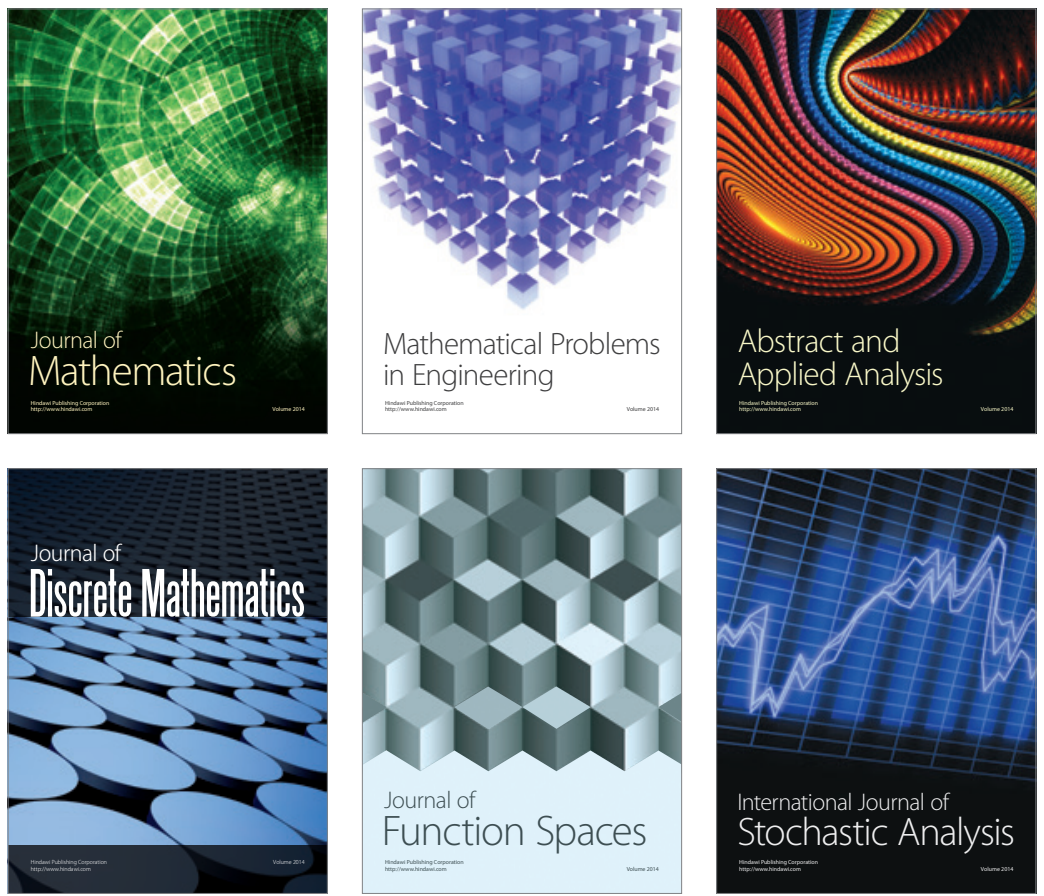

Journal of

Function Spaces

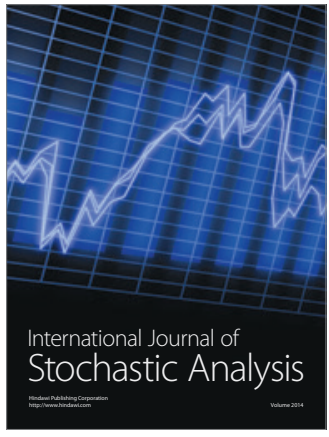

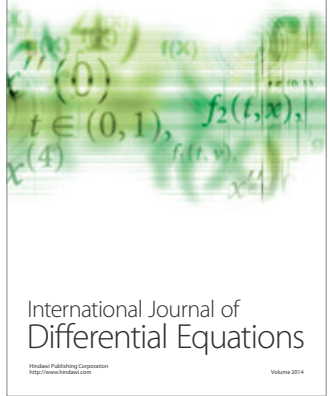
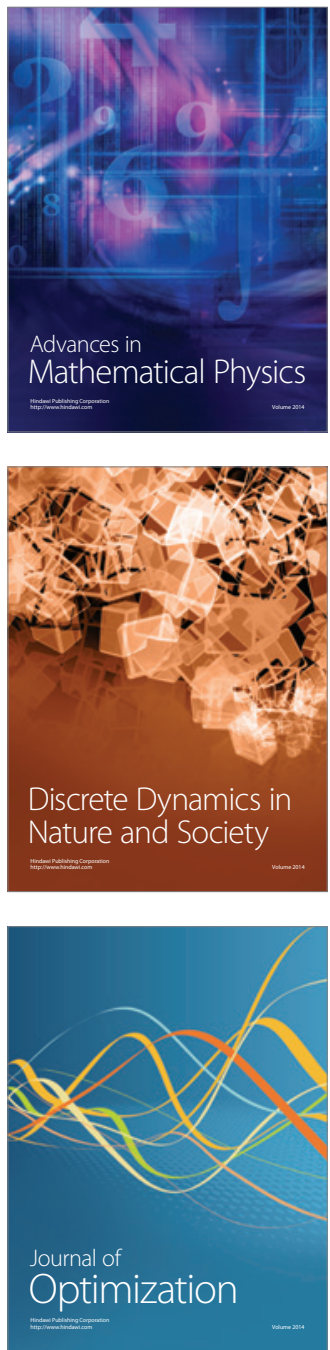